\title{
Modeling and Analysis of Malt-Drug Resistance Tuberculosis in Densly Populated Areas
}

\author{
Dancho Desaleng, Purnachandra Rao Koya* \\ School of Mathematical and Statistical Sciences Hawassa University, Hawassa, Ethiopia
}

Email address:

daniyabd9@gmail.com (D. Desaleng),drkpraocecc@yahoo.co.in (P. R. Koya)

\section{To cite this article:}

Dancho Desaleng, Purnachandra Rao Koya. Modeling and Analysis of Malt-Drug Resistance Tuberculosis in Densly Populated Areas. American Journal of Applied Mathematics. Vol. 4, No. 1, 2016, pp. 1-10. doi: 10.11648/j.ajam.20160401.11

\begin{abstract}
Tuberculosis is an airborne disease caused by the bacterium called mycobacterium tuberculosis. We have compartmentalized the population based on the exposed level to the disease and described the flow using a flowchart. Mathematical model is developed to describe the population dynamics of the compartments. The migration of people from infected class to exposed class, due to failure of continuing the medicine for any reason, is called here as Malt - drug resistance tuberculosis. The equilibrium points identified are disease free, endemic and epidemic. Equilibrium point analysis is made and has been included. Formula for reproduction number is derived. Numerical simulation study of the Mathematical model is conducted using ode45 function of MATLAB software. It is shown that the propagation of the disease is more in the more populated areas and less in the less populated areas.
\end{abstract}

Keywords: Tuberculosis, Mathematical Modeling, Equilibrium Points, Basic Reproduction Number, Stability Analysis, Numerical Simulation

\section{Introduction}

The word Mycobacterium is composed from the combination of two words viz., Myco and Bacterium. Myco means fungi and bacterium means unicellular micro organism. The meaning of Bacteria is a group of unicellular micro organisms. Bacteria are plural while bacterium is singular [1]. So far 137 mycobacterium species have been identified. The 'Mycobacterium tuberculosis complex' is one of 137 'mycobacterium species' which causes tuberculosis disease in human beings and animals. There are eight very closely related but different species in 'Mycobacterium tuberculosis complex' and they include Mycobacterium tuberculosis, Mycobacterium bovis, Mycobacterium africanum, Mycobacterium microti, Mycobacterium caprae, Mycobacterium pinnipedii, Mycobacterium Canetti and Mycobacterium mungi [2]. The bacteria called 'Mycobacterium bovis' causes tuberculosis disease in animals. Similarly, the bacteria called 'Mycobacterium tuberculosis' is a major cause for tuberculosis disease that affects human beings.

Tuberculosis is a bacterial air born infection disease and is caused by the Mycobacterium tuberculosis. The tuberculosis disease most commonly attacks the lungs of human beings.
The type of tuberculosis that attacks human lungs is known as 'pulmonary active tuberculosis'. Also, this variety of tuberculosis has the potential to spread to other organs such as bone and brain in the human body. Further, the tuberculosis can also affect the blood circulatory system, joints, central nervous system causing meningitis and even the skin. Whenever a person is infected with tuberculosis bacteria, the person first goes through a latent or noninfectious period. During the latent period the human body's immune system fights against the tuberculosis bacteria and at this stage Mycobacterium tuberculosis bacteria can not spread to the other human being [3]. The tuberculosis disease is characterized by the symptoms including cough for two up to three weeks or more, unexplained weight loss, fever, night sweats, loss of appetite, blood in the sputum or coughed up mucus etc [4].

The Mycobacterium tuberculosis bacterium is carried in airborne particles, called droplet nuclei, having a size of 1 5 microns in diameter. Infectious droplet nuclei are generated from the persons who have been suffering with pulmonary or laryngeal tuberculosis disease when they cough, sneeze, shout, sing and even when they just talk [5]. Depending on the environmental and climatically conditions, these tiny particles can remain suspended in the 
air for several hours. Transmission occurs when a person inhales droplet nuclei containing Mycobacterium tuberculosis. The droplet nuclei traverse through the mouth or nasal passages, upper respiratory track and then reach alveoli in the lungs. The transmission speed of the droplets depends on source patient, environment, exposure time and susceptible person. Repeated and close contacts with tuberculosis infected patient have been proved to be key and risky factors. An infected person who has not been treated for active tuberculosis disease will infect about 10 15 other persons per year [4].

The outbreak of infectious diseases causes mortality of millions of people. It also causes expenditure of enormous amount of money on health care and disease control activities. Many infectious diseases are directly resulted from poor hygienic conditions and repeated and close contact between infected and susceptible persons. Among the infectious diseases, Tuberculosis is proved to be the second largest killer disease. Tuberculosis contributes the adult mortality and it is one of serious health problems in today era. The deaths occurred worldwide during the years 2011, 2012 and 2013 are respectively $1.4,1.3$ and 1.5 millions $[5-7]$.

Over $95 \%$ of deaths occurred due to the tuberculosis disease take place in the 22 low and middle income countries mostly located in Sub Sahara Africa and in South East Asia continents. Sub-Sahara Africa carries the greatest proportion of new tuberculosis infected humans with over 260 cases per 100,000 people as per the records of 2011 [8]. Therefore, it is important that adequate attention is to be paid for preventing the spread of such diseases using effective control measures. Latent Tuberculosis can be diagnosed with a skin test called a Mantoux test. The test requires injecting PPD substance into the human body under the skin and examines after $48-72$ hours. This test is able to identify most of the people infected with tuberculosis bacilli within $6-8$ weeks after initial exposure. Active tuberculosis can be diagnosed by examining symptoms, x-rays, sputum and other samples collected from susceptible humans [9].

Tuberculosis is a very preventable disease. Providing adequate ventilation, opening of windows, improving conditions in the crowded places, infected people cover their mouth and nose when coughing or sneezing decreases the spread of tuberculosis. Identifying infected people in the early stages and treating them with proper medication is one of the most effective ways that helps to prevent tuberculosis from spreading. Some of the effective medications include the drugs Isoniazid (INH or $H$ ), Rifampin (RMP or R), Ethambutol (EMP or E) and Pyrazinamide (PZA or Z).The Isoniazid (INH) and Rifampin $(R M P)$ are first line drugs which have been given to the people who are infected with latent tuberculosis and who live in the risky areas or country side. If tuberculosis patients do not complete course of the treatment or do not take their medications as often as required, then a dangerous form of drug - resistant tuberculosis called Multi - Drug Resistant Tuberculosis (MDR-TB) occurs in the patient [9]. The purposes of the current study include (i) analysis of the spread of tuberculosis disease using mathematical model, (ii) evaluate the conditions at which disease will occur or die out using the basic reproduction number concept and (iii) multi-drug resistance stages of tuberculosis.

\section{Modeling and Formulations of Tuberculosis Disease}

Modeling of epidemics and diseases plays a very important role in developing prevention and controlling strategies. Mathematical models have been developed for the epidemics including Ebola, Brest cancer and Malaria [10 - 13]. Here in the present study we develop, analyze and make simulation study for Tuberculosis disease as an extension of the model given in [1].

The compartmental description and human flow directions of the model are illustrated in Figure 1. The model maintains the basic structure of the SEIR type models together with satisfying the following properties: (i) the recovered compartment $R$ is in the present study renamed as treatment compartment $T$ containing treated individuals. The patients of the treatment compartment $T$ are given medical treatment for the tuberculosis disease. But, after the treatment the individuals again may get exposed to tuberculosis disease and may enter the exposed compartment $E$, (ii) Mass action incidence rather than standard is assumed. That is, crowded or denser areas of populations are considered in this study, (iii) Treatment confers only partial immunity. That is, the tuberculosis disease cannot be cured completely with the medicines available as on today, (iv) Treated individuals are re infected at a rate $\lambda$ and with this rate people migrate from treated compartment $T$ to the exposed compartment $E$ and (v) Failure of treatment due to multi-drug resistance (MDR) of tuberculosis is considered. Thus, few people are expected to migrate from infected compartment $I$ to the exposed compartment $E$. Based on these assumptions the compartmental structure and flow directions of the model can be described using a directed flow chart as illustrated as follows:

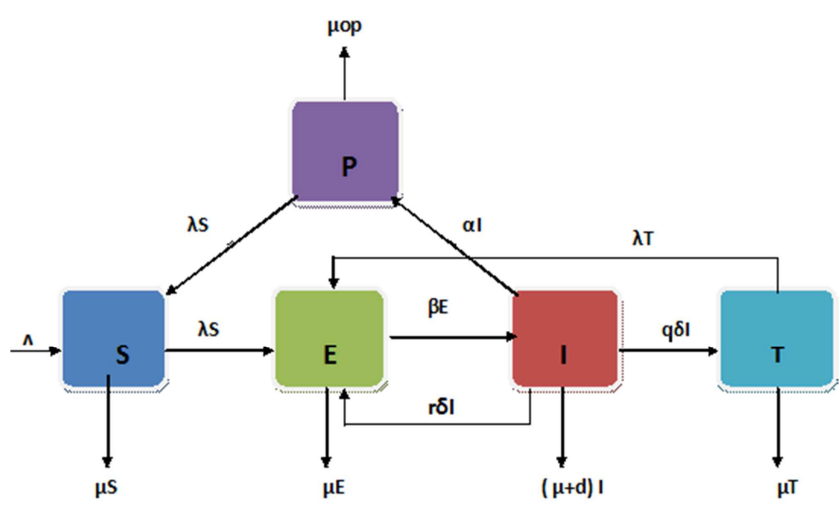

Figure 1. Flow chart of the compartmental model. 
The total human population $N_{H}(t)$ under study at any time $t$ is divided into four compartments. These four compartments are denoted by and they represent respectively $S(t)$ for susceptible, $E(t)$ for exposed but not infected, $I(t)$ for infected and $T(t)$ for treated but temporarily recovered people from the disease. Moreover, the total population is distributed into the four compartments and hence the sum of the populations of the four compartments is always the same as the total population. So that $N_{H}(t)=$ $S(t)+E(t)+I(t)+T(t)$ and all the variables of the relation are nonnegative. Also, all compartments contain and represent human beings. The concentration of small particles of Mycobacterium tuberculosis called tubercle bacilli in the air at any time $t$ is represented by $P(t)$.

Susceptible human population $S(t)$ We assume that the susceptible people are recruited from the total population at a constant rate $\Lambda$. Recruitment may occur as a result of new births and immigration from other regions. These susceptible individuals assumed to acquire Myco tuberculosis infection due to contact with the pathogen population at a rate $\lambda$. Here $\lambda=(\epsilon P / c)+P$. The exposed people are assumed to pollute the air at the rate $\epsilon$. The pathogen concentration of Myco tuberculosis in air is assumed to yield $50 \%$ and chance of catching the infection for a susceptible person is $c$. Finally, the susceptible population decreases by natural death at a rate of $\mu$. Thus, the rate of change of susceptible human population can be modeled by $d S / d t=\Lambda-(\lambda S / A) \mu S$.

Exposed human population the latently infected individuals are considered as not infectious because they do not to show any tuberculosis disease symptoms initially. The latently infected humans are not capable of transmitting bacteria to other susceptible humans. The population of the exposed individual is increased due to the reasons including newly infected individuals, exogenous re-infection or reactivation and failure of treatment. Exposed human population decreased by the progression of exposed individuals to active tuberculosis at a rate $\beta$ and natural death at a rate $\mu$. The rate of change of exposed compartment can be constructed as $d E / d t=[(\lambda S / A)+(\lambda T / A)+r \delta I-$ $(\beta+\mu) E]$.

Infected human population among all the exposed individuals, the tuberculosis disease will not develop in to the active tuberculosis disease as soon as the individuals are infected. Only about $10 \%$ of infected people become sick with tuberculosis after two or more years later and they will be then confirmed infected [1, 14]. The population of infected compartment increases at the rate $\beta$ due to the fast progress of the infection and development of the symptoms of tuberculosis disease. This infected population decreases at a rate $\delta$ because infected people recover temporarily from the disease after treatment. However, only a fraction $q$ of the medically treated infectious individuals are successful and migrate to the treated compartment. Unsuccessfully treated infected individuals re-enter the exposed compartment at rate $r=1-q$. Further, it is assumed that the natural death rate of infected individuals is $\mu$ and disease induced death rate is $d$. The rate of change of people in infected compartment can be constructed as $d I / d t=[\beta E-(\delta+$ $\mu+d) I]$

Treated human population treated individuals are assumed to recover from the illness only but not from tuberculosis disease. Tuberculosis infected individuals do not completely clear the bacteria from their body even after the treatment is successful. Instead they undergo a long latency period which could last many years or even a lifetime. The tuberculosis bacterium remains in the human body in a sleeping state [15]. Treated population increases at a rate of $\delta$ since the infected individuals temporarily recover from the disease by treatment. The treated population is decreased by exogenous re-infection or reactivation at the rate $\lambda$ and by natural death at the rate of $\mu$. The rate of change of population in the treated compartment can be constructed as $d T / d t=$ $\{q \delta I-[(\lambda / A)+\mu] T\}$.

Pathogen population in the polluted air In the high dense population areas like in the bus stations, inside buses, inside airplanes, in the camps, in the markets, in the schools, in the hospitals, in the homes, the environmental air may pollute with small particle of mycobacterium tuberculosis called tubercle bacilli. Infected individuals contribute to its enhancement through excretion of tuberculosis in to air at the rate $\alpha$. This small tuberculosis particle in the polluted air dies naturally at the rate $\mu_{0}$. The rate of change of Pathogen population in the polluted air compartment can be constructed as $(d P / d t)=\left(\alpha I-\mu_{0} P\right)$.

Thus, the system of non linear differential equations describing the dynamics of mycobacterium tuberculosis disease transmission model is given by

$$
\begin{aligned}
& (d S / d t)=\Lambda-[(\lambda / A)+\mu] S \\
& (d E / d t)=(\lambda / A)(S+T)+r \delta I-(\beta+\mu) E \\
& (d I / d t)=\beta E-(\delta+\mu+d) I \\
& (d T / d t)=q \delta I-(\mu+\lambda / A) T \\
& (d P / d t)=\left[\alpha I-\mu_{0} P\right]
\end{aligned}
$$

The variables and parameters used in the model equations (1) to (5) are described and interpreted in Table 1 and Table 2 respectively.

Invariant Region of the Model Variables Now on differentiating the total population variable $N_{H}(t)=S(t)+$ $E(t)+I(t)+T(t)$ with respect to time $t$ we get $N_{H}^{\prime}=$ $S^{\prime}(t)+E^{\prime}(t)+I^{\prime}(t)+T^{\prime}(t)$. The fore going expression takes the form as $N_{H}{ }^{\prime}(t)=\left(\Lambda-\mu N_{H}-d I\right)$ up on using the system of equations (1). However, the death rate of human beings due to the tuberculosis disease is very small and even closer to zero. Hence, without loss of generality we can choose this death rate to a very small positive quantity i.e. $\mathrm{d} \approx 0$. Therefore, the time differentiation of total population variable reduces to the expression $N_{H}{ }^{\prime}(t) \leq$ $\left[\Lambda-\mu N_{H}\right]$. On integrating this inequality using Birkhoff and Role's theorems we obtain the solution as $N_{H}(t) \leq$ $\left\{(\Lambda / \mu)-\left[(\Lambda / \mu)-N_{0}\right] e^{-\mu t}\right\}$. Here we have made use the condition that the initial human population size at $t=0$ is given by $N_{0}$, that is $N_{H}(0)=N_{0}$. It can be observed that as $t \rightarrow \infty$ the human population size $N_{H}(t)$ approaches the 
value $(\Lambda / \mu)$. Therefore, the feasible solution of human population for the model (1) is restricted to the region $\Omega_{H}=$ $\left\{(S, E, I, T) \in \mathbb{R}_{+}^{4}, N_{H}(t) \leq(\Lambda / \mu)\right\}$.

Similarly, from (1e) of the system of equations (1) the total Concentration of Mycobacterium tuberculosis in the environment denoted by $N_{p}$ reduces to $\left(d N_{P} / d t\right)=$ $\left[\alpha I-\mu_{0} P\right] \leq\left[\alpha N_{H}-\mu_{0} P\right]$, due to the reason $I(t) \leq$ $N_{H}(t) \leq(\Lambda / \mu)$. In view of these observations we can rewrite (1e) as $\left(d N_{P} / d t\right)=\alpha(\Lambda / \mu)-\mu_{0} N_{P}$ which is a first order ordinary linear differential equation having the particular solution $\mathrm{N}_{\mathrm{P}}(\mathrm{t}) \leq\left(\alpha \Lambda / \mu \mu_{0}\right)-\left[\left(\alpha \Lambda / \mu \mu_{0}\right)-\right.$ $\left.N_{0}\right] \mathrm{e}^{-\mu_{0} \mathrm{t}}$. Here we have assumed that the initial pathogen population is given by $N_{P}(0)=N_{0}$. Further it can be observed that as $t \rightarrow \infty$ the pathogen population size $N_{P}(t)$ satisfies the relation $0 \leq N_{P}(t) \leq\left(\alpha \Lambda / \mu \mu_{0}\right)$. Therefore, the feasible region of the pathogen population can be represented by the set $\Omega_{P}=\left\{N_{P} \in \mathbb{R}_{+}: N_{P} \leq\right.$ $\left.\left(\alpha \Lambda / \mu \mu_{0}\right)\right\}$.

Combining the feasible regions of both human $\Omega_{H}$ and pathogen populations $\Omega_{P}$ we obtain the feasible region of whole population $\Omega$ of the model (1) as $\quad \Omega=\left\{(S, E, I, T, P) \in \mathbb{R}_{+}^{5}:(S, E, I, T, P) \geq 0, N_{H} \leq\right.$ $(\Lambda / \mu)$ and $\left.N_{P} \leq\left(\alpha \Lambda / \mu \mu_{0}\right)\right\}$.

Recall that the variables $N_{H}=(S+E+I+T)$ and $N_{P}=$ $P$ represent the population sizes of humans and pathogens respectively. Further, it can be verified that $\Omega$ is positively invariable set induced by the system of equations (1). Hence the system (1) is biologically meaningful and mathematically well-posed with in the domain given by the region $\Omega$. Thus, it is feasible to consider the dynamics and flow of human and pathogen populations as described by the model (1) with in the region $\Omega$.

Table 1. Description of parameters used in the model equations (1) to (5).

\begin{tabular}{ll}
\hline Variables & Description \\
\hline$S(t)$ & Human population size of susceptible compartment at time $t$ \\
$E(t)$ & Human population size of exposed compartment at time $t$ \\
$I(t)$ & Human population size of infected compartment at any time $t$ \\
$T(t)$ & Human population size of treated compartment at any time $t$ \\
$P(t)$ & $\begin{array}{l}\text { Mycobacterium tuberculosis concentration in the } \\
\text { environment at any time } t\end{array}$ \\
$N_{H}(t)$ & Total human population size at any time $t N_{H}(t)=S(t)+$ \\
\hline
\end{tabular}

Table 2. Notations and description of the model parameters used in (1) to (5).

\begin{tabular}{ll}
\hline Parameter & Description \\
\hline$\Lambda 1$ & $\begin{array}{l}\text { Recruitment rate by birth and immigration of humans into } \\
\text { susceptible compartment }\end{array}$ \\
$\beta$ & $\begin{array}{l}\text { Progression rate of humans from exposed compartment to } \\
\text { infected compartment }\end{array}$ \\
$\epsilon$ & $\begin{array}{l}\text { Exposure rate of susceptible human to polluted air } \\
\text { Rate of infected individual is treated } \\
\text { Concentration amount of mycobacterium tuberculosis in the } \\
\text { air that yields more than } 50 \% \text { chance of infecting }\end{array}$ \\
$c$ & $\begin{array}{l}\text { susceptible individual } \\
\text { Part of the area occupied by humans in a square meter }\end{array}$ \\
$\mu$ & $\begin{array}{l}\text { Per-capita natural death rate for humans in any compartment } \\
\text { Contribution by each infected individual to the population }\end{array}$ \\
$\alpha$ & size of Mycobacterium tuberculosis in the air \\
$r$ & Success rate of treatment \\
\hline
\end{tabular}

\section{Stability Analysis of the Model}

In this section, we (i) identify the existence of equilibrium points viz. disease free equilibrium point, endemic equilibrium point and epidemic equilibrium point (ii) analyze local stability of disease free equilibrium point and (iii) construct the formula for reproduction number.

\subsection{Existence of Equilibrium Points}

The equilibrium points are obtained by setting the righthand sides of the system equation of model (1) to zero. That means $(d S / d t)=(d E / d t)=(d I / d t)=(d T / d t)=$ $(d P / d t)=0$. The foregoing condition is a requirement for existence of equilibrium points.

\subsection{The Disease Free Equilibrium Point}

Disease-free equilibrium point denoted by $x_{0}$ is a steadystate solution when there is no tuberculosis disease. The human populations of exposed, infected and treatment compartments can be considered as tuberculosis infected. Also in absence of infection the exposed, infected, treatment compartments of human population and pathogen compartment of bacterium population are empty. That is $E^{*}(t)=I^{*}(t)=T^{*}(t)=P^{*}(t)=0$. Here the variables $E^{*}(t), I^{*}(t)$ and $T^{*}(t)$ respectively represent the human population sizes of exposed, infected and treatment compartments when there is no disease. Also $S^{*}(t)$ represents total human population size when there is no disease. Similarly, $P^{*}(t)$ represents the pathogen population in the air when there is no disease. But the equation (1a) at equilibrium point reduces to $\left[\Lambda-\left(\lambda S^{*} / A\right)-\mu S^{*}\right]=0$ and up on substituting the value $\lambda=[\epsilon P /(c+P)]$ we obtain $S^{*}=$ $(\Lambda / \mu)$. Therefore the mycobacterium tuberculosis disease free equilibrium point is given by $x_{0}=\left(S^{*}, E^{*}, I^{*}, R^{*}, P^{*}\right)=$ $\{(\Lambda / \mu), 0,0,0,0\}$.

\subsection{Reproduction Number}

The basic reproduction number denoted by $R_{0}$ is defined as the average number of secondary infections caused by an infectious individual during his or her entire period of infectiousness. It is an important parameter in epidemiology as it sets the threshold in the study of a disease both for predicting its outbreak as well as evaluating its control strategies. Persistent and die out of the disease in a community is depend on the size of the reproduction number $R_{0}$. Now, the basic reproduction number $R_{0}$ will be found by using the method of next generation matrix [16].

Suppose there are $n$ and $m$ compartments containing populations with disease and non-disease respectively. Let $x \in \mathbb{R}^{n}$ and $y \in \mathbb{R}^{m}$ be the total sub-population sizes of these compartments. The compartmental model can then be expressed as $x_{i}^{\prime}=\left[f_{i}(x, y)-v_{i}(x, y)\right], \forall i=1, \ldots, n$ and $y_{j}^{\prime}=\left[g_{j}(x, y)\right], \forall j=1, \ldots, m$. Here the upper prime denotes differentiation with respect to time $t$. Note that the decomposition of the dynamics into $f$ and $v$ and the designation of compartments as infected or uninfected may not be unique. But, different decompositions are possible 
correspond to different epidemiological diseases and interpretations of models.

Let us now consider the following notations and their representations: (i) $f_{i}(x)$ denotes the rate of appearance of new infection cases in $i^{\text {th }}$ compartment, (ii) $v_{i}^{+}$denotes the transfer rate of individuals into $i^{\text {th }}$ compartment by all means (iii) $v_{i}^{-}$denotes the transfer rate of individuals out from $i^{\text {th }}$ compartment by all means and (iv) we also denote $v_{i}=$ $v_{i}^{-}-v_{i}^{+}$. Further, it is assumed that each of the three functions $f_{i}(x), v_{i}^{+}$and $v_{i}^{-}$is continuously differentiable for at least two times with respect to their arguments.

Let us now define two matrices $F$ and $V$ by $F=$ $\left[\left(\partial / \partial x_{j}\right) f_{i}\left(x_{0}\right)\right]$ and $V=\left[\left(\partial / \partial x_{j}\right) v_{i}\left(x_{0}\right)\right]$ respectively. Here the number of compartments denoted by $i$ satisfies the condition $i \geq 1$ while the number of infected compartments denoted by $j$ satisfies the condition $1 \leq j \leq n$. Then the matrix $F V^{-1}$ is referred to as the next generation matrix for the system of model equations (1) at the disease free equilibrium point.

Also the reproduction number is defined in terms of next generation matrix as $R_{0}=\rho\left(F V^{-1}\right)$. Here $\rho(A)$ denotes the spectral radius or magnitude of the largest eigenvalue of the matrix $A$. Also, (i) the matrix of the new infection terms denoted by $F$ and (ii) the non singular matrixof the remaining transfer terms denoted by $V$ are both $n \times$ $n$ matrices. Here $n$ is the number of infected compartments. Note that the elements of the matrix $F$ are non-negative. The $(i, j)$ element of the matrix $F$ represents the rate at which infected individuals transfer from $j^{\text {th }}$ compartment to $i^{\text {th }}$ compartment.

Consider that an infected individual is transferred into a disease free compartment $k$. The $(j, k)$ entry of the matrix $V^{-1}$ represents the average time an infected individual spends in compartment $j$ during its lifetime, assuming that the population remains near the disease free equilibrium point and barring re-infection.

Hence, the $(i, k)$ entry of the product matrix $\mathrm{FV}^{-1}$ gives the expected number of new infections in compartment is produced by the infected individual originally introduced into compartment $k$. Using the next - generation approach and taking the infected compartments to be $E, I$ and $P$ from system of equations (1) gives $(d E / d t)=[\lambda S / A+\lambda T / A+$ $r \delta I-(\beta+\mu) E] \quad, \quad(d I / d t)=[\beta E-(\delta+\mu+d) I]$ and $(d P / d t)=\left[\alpha \mathrm{I}-\mu_{0} \mathrm{P}\right]$. Here the progression rate from exposed compartment to infected compartment and the failure rate of treatment are not considered as new infection cases. From this we define $f_{i}$ and $v_{i}$ as

$$
f_{i}=\left[\begin{array}{c}
\frac{\epsilon P S}{A(c+P)}+\frac{\epsilon P T}{A(c+P)} \\
0 \\
0
\end{array}\right], v_{i}=\left[\begin{array}{c}
(\beta+\mu) E-r \delta I \\
(\delta+\mu+d) I-\beta E \\
\mu_{0} P-\alpha I
\end{array}\right]
$$

Now partially differentiating the variables $E, I$ and $P$ with respect to time and evaluating at the disease free equilibrium point reduces the Jacobian matrices to

$$
\mathrm{F}=\left[\begin{array}{ccc}
0 & 0 & (\in \Lambda / \mathrm{cA} \mu) \\
0 & 0 & 0 \\
0 & 0 & 0
\end{array}\right], \mathrm{V}=\left[\begin{array}{ccc}
\beta+\mu & -\mathrm{r} \delta & 0 \\
-\beta & \delta+\mu+\mathrm{d} & 0 \\
0 & -\alpha & \mu_{0}
\end{array}\right]
$$

Finding the inverse of the Jacobian matrix of $V$ and computing the product of both matrices $F$ and $V^{-1}$ we get

$$
\left(\mathrm{FV}^{-1}\right)=\left[\begin{array}{ccc}
\frac{\alpha \in \beta \Lambda}{\xi(\beta+\mu)-\mathrm{r} \beta \alpha} & \frac{\alpha \in \Lambda}{\xi-\mathrm{r} \beta \alpha} & \frac{\epsilon \Lambda}{\mathrm{Ac} \mu \mu_{0}} \\
0 & 0 & 0 \\
0 & 0 & 0
\end{array}\right]
$$

Here $\xi=\left[\right.$ A c $\left.\mu \mu_{0}(\delta+\mu+\mathrm{d})\right]$.

The eigenvalues of the matrix $\left(F V^{-1}\right)$ are the solutions of the characteristic equation $G=\left|F V^{-1}-I \lambda\right|=0$, where $\mathrm{I}$ is identity matrix. The solutions are found to be $\lambda_{1}=$ $\{[\alpha \in \Lambda \beta] /[\xi(\beta+\mu)-\mathrm{r} \beta \alpha]\}, \quad \lambda_{2}=0$ and $\lambda_{3}=0$. It is well known that the dominant eigenvalue is considered to be the basic reproduction number $R_{0}$ of the model. Therefore, it can be seen clearly that $\lambda_{1}$ is the largest eigenvalue. Thus, the reproduction number is given by

$$
R_{0}=\left[\frac{\alpha \epsilon \Lambda \beta}{A c \mu \mu_{0}(\beta+\mu)(\delta+\mu+d)-r \beta \alpha}\right]
$$

\subsection{Local Stability of the Disease Free Equilibrium Point}

Under the local stability of the disease free equilibrium point we try to explain three conditions. Condition 1 The disease free equilibrium point $x_{0}$ is said to be locally asymptotically stable if the real parts of the eigenvalues are all negative and unstable if the real part of the eigenvalues are positive. Condition 2 If $\mathrm{R}_{0}<1$, then disease free equilibrium point $x_{0}$ is locally asymptotically stable i.e. no tuberculosis epidemic can develop in the population and if $\mathrm{R}_{0} \geq 1$, disease free equilibrium point $x_{0}$ is unstable i.e. tuberculosis epidemic and endemic can develop in the population. Condition 3 Using the Routh-Hurwitz method. The Routh method is used for determining the stability of linear continuous data system without involving root solving.

The local stability of the disease free equilibrium point $x_{0}=\{(\Lambda / \mu), 0,0,0,0\}$ can be analyzed by computing the Jacobian matrix of the model equations (1) to (5). On differentiating each equation of the system (1) to (5) with respect to $S, E, I, R$ and $P$ and evaluating the resultants at the disease free equilibrium point $\mathrm{S}=(\Lambda / \mu), \mathrm{E}=0, \mathrm{I}=0, \mathrm{~T}=$ 0 and $\mathrm{P}=0$ we get

$$
J\left(x_{0}\right)=\left[\begin{array}{ccccc}
-\mu & 0 & 0 & 0 & -\phi \\
0 & -\gamma & r \delta & 0 & \phi \\
0 & \beta & -\psi & 0 & 0 \\
0 & 0 & q \delta & -\mu & 0 \\
0 & 0 & \alpha & 0 & -\mu_{0}
\end{array}\right]
$$

Here in (10), $\psi=(\delta+\mu+d), \phi=[\epsilon \Lambda / A c \mu]$ and $\gamma=$ $(\beta+\mu)$.

Consider the matrix (3) and let $\kappa$ be the eigenvalue of the characteristic equation $\left|J\left(x_{0}\right)-\kappa I\right|=0$, where $I$ is an $5 \times 5$ identity matrix. Thus, we have

$$
\left|J\left(x_{0}\right)-\kappa I\right|=\left|\begin{array}{ccccc}
-\mu-\kappa & 0 & 0 & 0 & -\phi \\
0 & -\gamma-\kappa & r \delta & 0 & \phi \\
0 & \beta & -\psi-\kappa & 0 & 0 \\
0 & 0 & q \delta & -\mu-\kappa & 0 \\
0 & 0 & \alpha & 0 & -\mu_{0}-\kappa
\end{array}\right|
$$


As the first and fourth columns correspond to the total human populations and contain only the diagonal terms, these diagonal terms form two similar eigenvalues of the Jacobian matrix (11). Thus, setting $[-(\mu+\kappa)]=0$ implies that $\kappa_{1}=\kappa_{4}=(-\mu)$. This is in accordance with Condition 1 stating that $x_{0}$ is stabile.

The other three eigenvalues are the roots of the characteristic equation of the matrix formed by excluding the first and fourth rows and first and fourth columns of the Jacobian matrix (11), we obtain the resultant sub matrix as

$$
\left|J^{*}\left(x_{0}\right)-k I\right|=\left|\begin{array}{ccc}
-\gamma-\kappa & \gamma \delta & \phi \\
\beta & -\psi-\kappa & 0 \\
0 & \alpha & \mu_{0}-\kappa
\end{array}\right|
$$

Now, up on substituting the values of $\gamma, \phi$ and $\psi$ and evaluating the Jacobian determinant (12) we get the characteristic equation as $[(\beta+\mu+\kappa)(\delta+\mu+d+\kappa)(\mu+$ $\left.\kappa)-r \delta \beta\left(\mu_{0}+\kappa\right)-\varepsilon \alpha \beta \Lambda / A c \mu\right]=0 \quad$. Also, the characteristic equation can be expressed as

$$
a_{3} \kappa^{3}+a_{2} \kappa^{2}+a_{1} \kappa+a_{0}=0
$$

In (13), we have used the notations $a_{3}=1, a_{2}=$ $\left[2 \mu+\mu_{0}+\beta+\delta+d\right] \quad, \quad a_{1}=\left[\mu_{0}(2 \mu+\beta+\delta+d)+\right.$ $(\beta+\mu)(\mu+\delta+d)-r \delta \beta]$ and $a_{0}=\left[\mu_{0}[(\beta+\mu)(\mu+\delta+\right.$ $d)-r \delta \beta]-\epsilon \alpha \beta \Lambda / c A \mu]$. Due to the complexity in determining the signs of the remaining three eigenvalues of (13), we now employ the Routh - Hurwitz conditions for stability. The Routh - Hurwitz criteria for the polynomials of order three summarized that Row $3=\left[\begin{array}{ll}a_{3} & a_{1}\end{array}\right]$, Row $2=$ $\left[\begin{array}{ll}a_{2} & a_{0}\end{array}\right]$, Row $1=\left[\begin{array}{ll}b_{1} & 0\end{array}\right]$ and Row $0=\left[\begin{array}{ll}c_{1} & 0\end{array}\right]$. Here $b_{1}=\left(\frac{-1}{a_{2}}\right)\left|\begin{array}{ll}a_{3} & a_{1} \\ a_{2} & a_{0}\end{array}\right| \quad$ and $\quad c_{1}=\left(\frac{-1}{b_{1}}\right)\left|\begin{array}{cc}a_{2} & a_{0} \\ b_{1} & 0\end{array}\right|=a_{0}$ Therefore, if $a_{3}>0$, necessary and sufficient condition for third order system to be stable is that $a_{2}>0, b_{1}>0$ and $a_{0}>0$. Clearly from these expressions we observe that $a_{3}$ and $a_{2}$ are positive quantities. Again we have that $b_{1}>$ 0 is equivalent to $a_{2} a_{1}>a_{0} a_{3}$ and this implies that $a_{1}>0$. Therefore $a_{3}>0, a_{2}>0$ and $a_{1}>0$ and this implies that $x_{0}$ is stabile.

We now consider that the quantity $a_{0}$ isa positive. That is, $\quad a_{0}>0 \quad$ simplifies $\left\{1-\left\{(\epsilon \alpha \Lambda \beta) /\left\{A c \mu \mu_{0}[(\beta+\mu)(\mu+\delta+d)-r \delta \beta]\right\}\right\}\right\}>0$. This implies that $\left(1-R_{0}\right)>0$ or equivalently $R_{0}<1$. Hence, according Condition 2 the disease free equilibrium point $x_{0}$ is stabile. Thus, we conclude that the disease free equilibrium point $x_{0}$ is locally stable.

\subsection{Endemic and Epidemic Equilibrium Points}

Endemic and Epidemic equilibrium points, $x_{1}$ is a steady state solution, where the disease persists in the population. The existence and uniqueness of endemic and epidemic equilibrium points $x_{1}$ is should satisfy the conditions: $x_{1}=$ $\left(S^{*}, E^{*}, I^{*}, R^{*}, P^{*}\right) \neq 0$ and $x_{1}=\left(S^{*}, E^{*}, I^{*}, R^{*}, P^{*}\right)>0$. Setting right-hand side zero for the third equation of the system of equations (1) and rewriting for $I^{*}$, we get

$$
I^{*}=\left[\beta E^{*} /(\delta+\mu+d)\right] .
$$

Again taking the second equation of the system (1) and setting right-hand side zero. Finally, we evaluate for the exposed compartment $E^{*}$ as

$$
E^{*}=\left\{\lambda\left(S^{*}+T^{*}\right) /[A(\beta+\mu)]\right\}+\left[r \delta I^{*} /(\beta+\mu)\right]
$$

First equation from the system of equations (1) to (5) evaluated by setting the right-hand side zero and writing with respect to $S^{*}$ as

$$
S^{*}=\{\Lambda /[(\lambda / A)+\mu]\}
$$

The fourth equation from the system of equations (1) to (5) also evaluated by setting the right-hand side zero and writing with respect to $T^{*}$ as

$$
T^{*}=\left\{\Lambda \mathrm{q} \delta \mathrm{I}^{*} /[(\lambda / A)+\mu]\right\}
$$

Then substituting equations (16) and (17) in to equation (15) we get

$$
E^{*}=(\tau / \omega)+\left[r \delta I^{*} /(\beta+\mu)\right]
$$

Here in (18), we have used the notations $\tau=[A \lambda(\Lambda+$ $\left.q \delta I^{*}\right]$ and $\omega=[A(\beta+\mu)(\lambda+A \mu)]$.

Then substituting equation (18) in to equation (14) finally we get

$$
I^{*}=\{(A \Lambda \lambda \beta) /[\varphi-\sigma-\theta]\}
$$

Here in (19), we have used the notations $\varphi=[A(\mu+$ $\beta)(\delta+\mu+d)(\lambda+A \mu)] \quad, \quad \sigma=[q \delta \beta A \lambda] \quad$ and $\theta=$ $[r \delta \beta A(\lambda+A \mu)]$. But from the equation (5) we can evaluate $P^{*}$ by setting the right-hand side zero as

$$
P^{*}=\left(\alpha I^{*} / \mu_{0}\right)
$$

Then substituting equation (19) in to equation (20)

$$
P^{*}=\left\{(\alpha A \Lambda \lambda \beta) /\left(\mu_{0} \varphi-\mu_{0} \sigma-\mu_{0} \theta\right]\right\}
$$

Furthermore, we have $\lambda=\left[\epsilon P^{*} /\left(c+P^{*}\right)\right]$ and substituting the value of $P^{*}$ from (21) $\lambda$ takes the expression $\lambda=\left[(\epsilon \alpha A \Lambda \lambda \beta) /\left[c \mu_{0} \varphi-c \mu_{0} \sigma-c \mu_{0} \theta+\alpha A \Lambda \lambda \beta\right]\right]$. Also on substituting the values of $\varphi, \sigma, \theta$ and after crossmultiplication and simplification the implicit expression for $\lambda$ can be expressed as

$$
M \lambda^{2}+X \lambda=0
$$

In (22), $M=c A \mu_{0}[(\beta+\mu)(\delta+\mu+d)-r \delta \beta-q \delta \beta]-$ $\alpha A \beta \Lambda$ and $X=A^{2} c \mu \mu_{0}[(\beta+\mu)(\delta+\mu+d)-r \delta \beta]-$ $\alpha \in A \beta \Lambda$. Clearly from equation (22) one of the solutions is $\lambda_{1}=0$ and this corresponds to the disease free equilibrium point $x_{0}$.

\subsubsection{The Epidemic Equilibrium Point}

Epidemic means widespread outbreak of an infectious disease. People more in number are expected to be infected at same time. Spread of the tuberculosis disease over a considerable extent of population is computed by using $X$ value. Clearly $M$ is a positive quantity. Further, if $X$ is also a positive quantity then $M \lambda+X=0$ has negative solution $\lambda=-(X / M)$. But, a negative value 
for $\lambda$ is not desirable. Hence, positive value for $X$ is also not desirable. So we consider that $X$ is a negative quantity i.e. $X<0$.This consideration, after some simplifications, leads to the result $R_{0}>1$. Therefore if $X<0$ or equivalently $R_{0}>1$ thereexists a positive solution for (22). The biological meaning of $X<0$ or equivalently $R_{0}>1$ is that the tuberculosis disease spread continues in the society. One infected person can transmit disease to more than one person.

\subsubsection{The Endemic Equilibrium Point}

Tuberculosis is a kind of endemic disease that constantly presents with a greater or lesser degree among the people of certain class or among the people living in a particular location. From quadratic equation (22) can be simplified to give $M \lambda+X=0$ and this corresponds to the existence of an endemic equilibrium point. The critically looking at the quadratic equation (22) clearly shows that there is an endemic equilibrium point exists. The equation (22) represents an upper parabola and essentially it has a minim point when $M>0$.

The existence of the unique endemic equilibrium point requires that the discriminate of quadratic equation (22) is zero. That is $X^{2}-4 M C=0$. But in the present case $C=$ 0 and hence we get $X^{2}=0$.This result implies that $\left.x^{2}=\mathrm{Z}-\alpha \in A \beta \Lambda\right)^{2}=0$ $\left[(-\alpha \epsilon A \beta \Lambda)^{2}(1-(\mathrm{Z} / \alpha \epsilon A \beta \Lambda))^{2}\right]=0$ or $\left[(-\alpha \epsilon A \beta \Lambda)^{2}(1-\right.$ $\left.\left.1 / R_{0}\right)^{2}\right]=0$ or $\left[\left(\alpha \in A \beta \Lambda / R_{0}\right)^{2}\left(1-R_{0}\right)^{2}\right]=0$ or $X_{1}^{2}(1-$ $\left.R_{0}\right)^{2}=0$. Here $\mathrm{Z}=\left(A^{2} c \mu \mu_{0}[(\beta+\mu)(\delta+\mu+d)-r \delta \beta]\right.$ and $X_{1}=\left(\alpha \in A \beta \Lambda / R_{0}\right)$ thus $\left(1-R_{0}\right)^{2}=0$.The solution is $R_{0}=1$. The biological meaning of $R_{0}=1$ is that one infected person during his life time can transmit disease to only one new person. The endemic and epidemic points can be summarized as (i) Endemic equilibrium point exist if $R_{0}=1$ and (ii) Epidemic equilibrium point exists if $R_{0}>1$.

\section{Numerical Simulations}

Numerical Simulations of the model equations (1) to (5) were carried out using MATLAB inbuilt function ode 45 Runge - Kutta of order four. To control the spread of tuberculosis disease the selecting parameters play an important role. Hence, we have to focus more on the selection of parameters values. Most of the parametric values used here are collected from literature and the remaining are all based on our assumptions. Further five hypothetical cases were considered with variable size of area $A$ as $0.20,0.90,2$, 20 and 200 in square meters. And the initial conditions have been considered as $\mathrm{S}[0]=1600 ; \mathrm{E}[0]=1500 ; \mathrm{I}[0]=150 ; \mathrm{R}[0]=$ $140 ; \mathrm{P}[0]=100$ at initial time $t_{0}=0$. Also the final time is considered as $t_{f}=10$. The parametric values are considered based literature $[17,18]$ and mainly on assumptions as: $\Lambda=$ $0.02 * 10^{6}, \mu=0.02, \beta=0.352, \delta=0.99, q=0.45, d=$ $0.365, \alpha=0.2, \epsilon=0.1, \mu_{0}=0.99$ and $c=80$. The results of the simulation study are presented in the following figures.

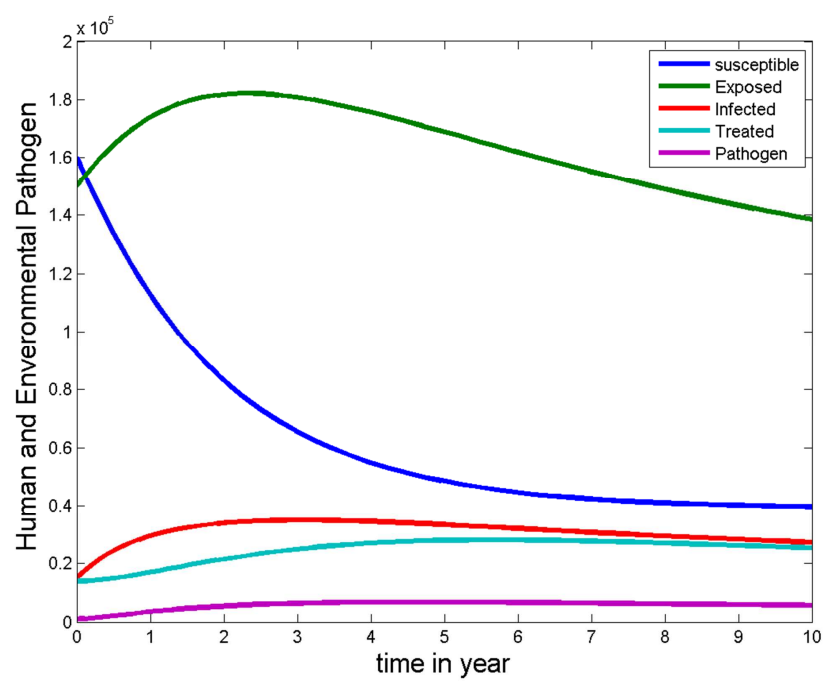

Figure 2. Reproduction ratio $R_{0}=868.9041$ and force of contact rate $\lambda=0.0992$ at $A=0.2$ square meter.

In Figure 2 we see that population size of susceptible compartment decreases with time and finally reach a positive lower asymptote. The population sizes of all the remaining compartments viz., exposed, infected, treated and pathogen will increase and reach their corresponding upper asymptotes. Since, the reproduction ratio is greater than one the spread of tuberculosis is very high in population.

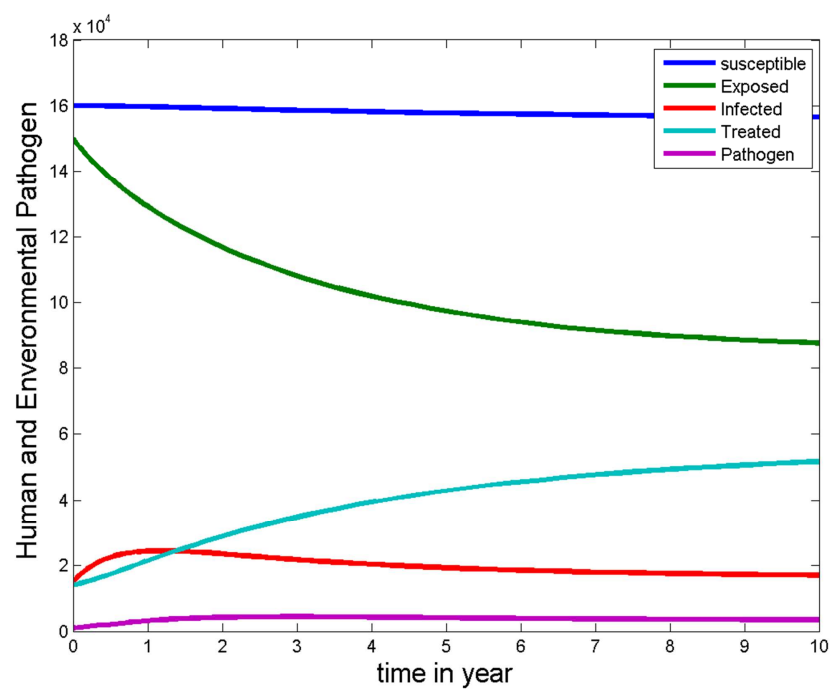

Figure 3. Reproduction ratio $R_{0}=193.0898$ and force of contact rate $\lambda=0.0986$ at area $A=0.9$ square meter .

In Figure 3 we observe that the population size of susceptible compartment increases with progression of time. The population sizes of exposed decreases and reaches its lower asymptote. The population sizes of infected, treated and pathogen increase and reach their upper asymptotes. Still the spread of tuberculosis disease occurs in the population because the reproduction ratio is greater than one. 


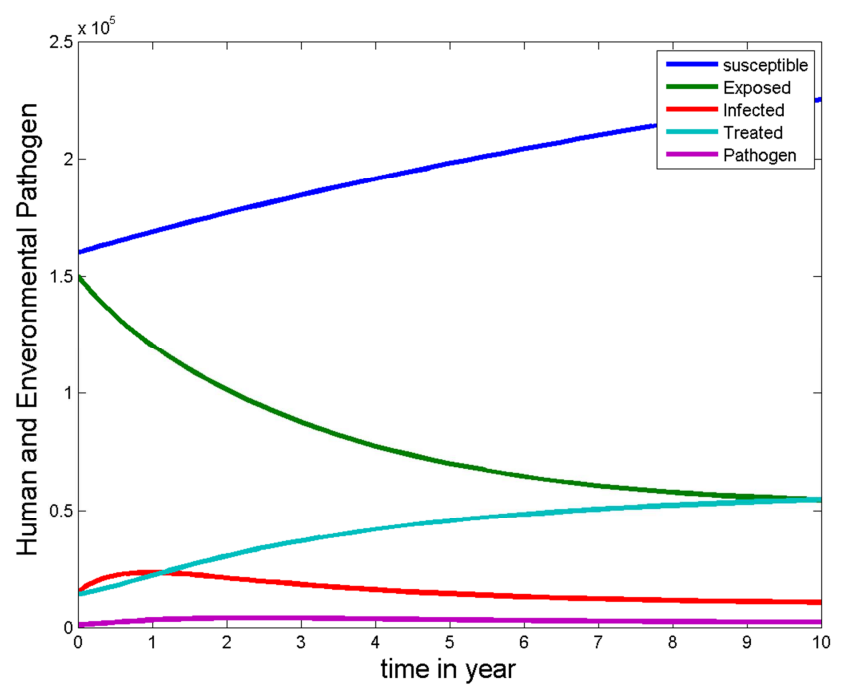

Figure 4. Reproduction ratio $R_{0}=86.8904$ and force of contact rate $\lambda=0.0978$ in the area of $A=2$ square meters.

In Figure 4 we see that, the population sizes of susceptible and treated compartments increase with progression of time. The population sizes of all the remaining compartments viz., exposed, infected, and pathogen will decrease. The reproduction ratio is greater than one and also the widespread of tuberculosis disease in population increases.

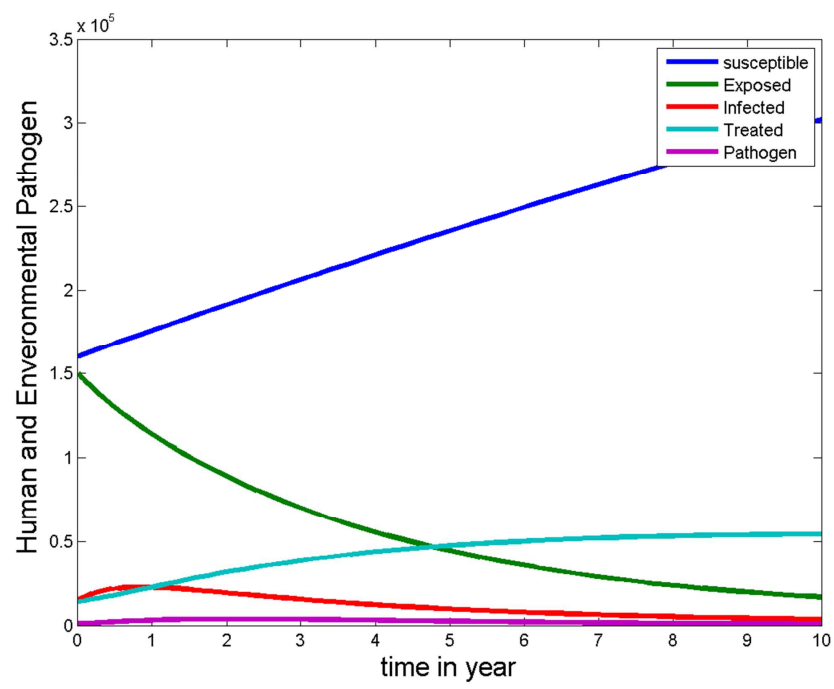

Figure 5. Reproduction ratio $R_{0}=8.6890$ and force of contact rate $\lambda=0.0952$ at area of $A=20$ square meter.

From the figure population sizes of susceptible and treated compartment increase with progression of time. The population sizes of exposed, infected and pathogen compartments decrease and ultimately converge to zero as time takes larger values. The population sizes of the infected and recovered compartments will increase initially and at later times decrease and converge to zero. But here the reproduction ratio is greater than one. Therefore one tuberculosis infected patient can transmit disease to more than one new uninfected.

Figure 6 shows that the population sizes of susceptible and treated compartments increase with progression of time. The population sizes of exposed and pathogen compartments decrease and ultimately converges to zero as time takes larger values. The population size of the infected compartment will increase initially and at later times decrease and converge to zero finally. Here, the reproduction ratio is less than one. Therefore there is no tuberculosis disease spread in the population.

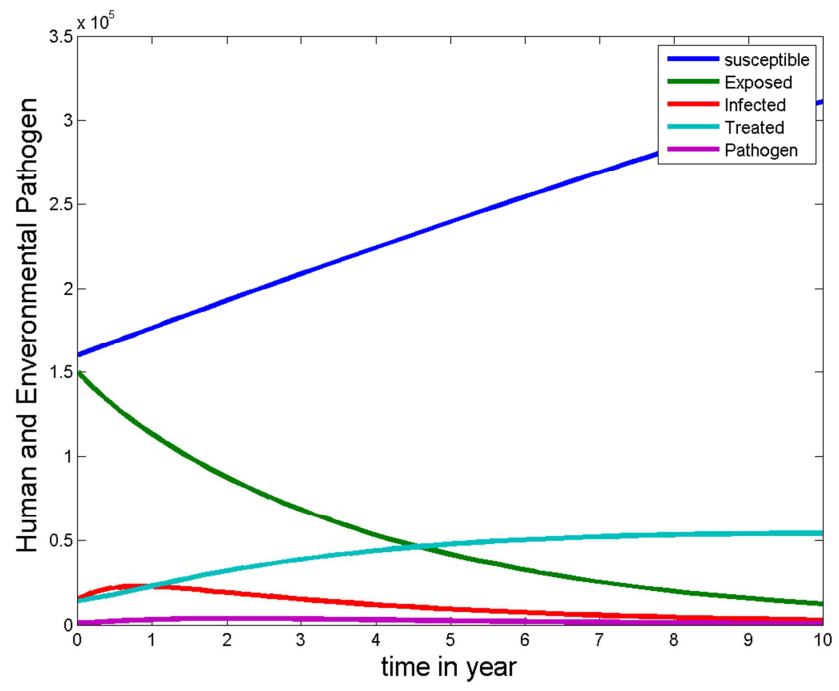

Figure 6. Reproduction ratio $R_{0}=0.8689$ and force of contact rate $\lambda=0.0944$ in the area of $A=200$ square meters.

\section{Comparison of the Models}

Here under we present the comparisons of the present work with our previous work [1].

When compare both reproduction ratios of the present and previous [1] study the area of size 2 square meters we get the clear difference and the same is shown in Figure 7.

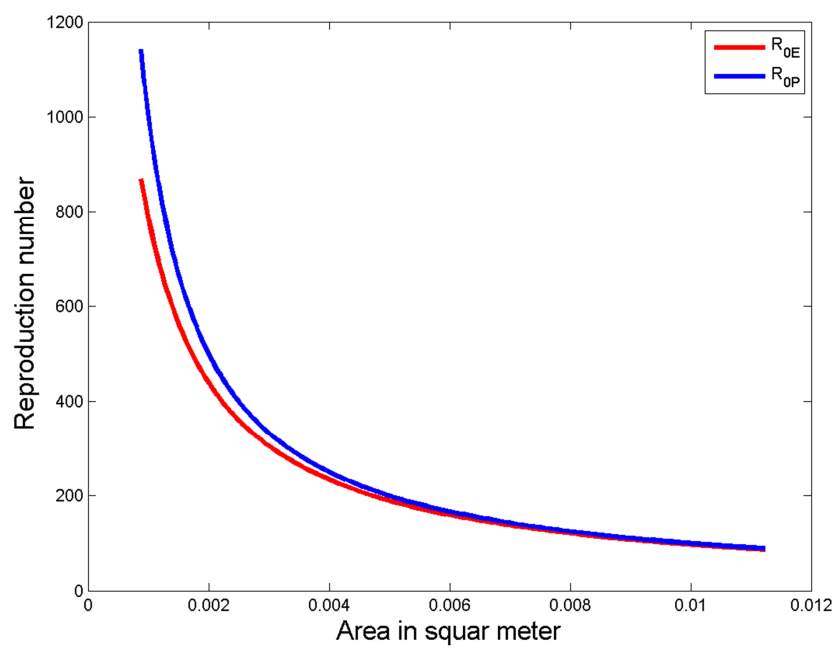

Figure 7. Comparison of $R_{0}$ of the present and earlier studies.

Here we represent the reproduction ration of the present study by $R_{0 P}$ and that of the earlier study by $R_{0 E}$. If size of the area increases in square meters then the reproduction ratio $R_{0 P}$ decreases fast and coincides with reproduction 
ratio $R_{0 E}$. If size of the area approaches to zero then the value of reproduction ratio $R_{0 P}$ become larger than reproduction ratio $R_{0 E}$. Therefore reproduction ratio $R_{0 P}$ is faster than $R_{0 E}$ to attack a suspected people in very crowded area. This means when one tuberculosis patient presents in a community and the community lives together in a very crowded area then the tuberculosis patient transmits disease to many susceptible people within a short period of time.

Table 3. Comparison of the results of previous and present studies.

\begin{tabular}{|c|c|c|}
\hline S. No. & Results of previous study [1] & Results of present study \\
\hline 1 & The total human population is divided into four compartments. & The total human population is divided into four compartments. \\
\hline 2 & The model has SEIR structure. & $\begin{array}{l}\text { The model has } S E I R \text { structure. But } R \text { compartment is replaced by a } \\
\text { compartment of treated individuals } T\end{array}$ \\
\hline 3 & Specific area was assumed & Specific area was assumed \\
\hline 4 & Treatment is given only to the people of infected compartment & Treatment is given only to the people of infected compartment \\
\hline 5 & Treatment confers only partial immunity & Treatment confers only partial immunity \\
\hline 6 & Recovery indicates recovery from the illness but not from the disease. & Treated indicates recovery from the illness but not from the disease \\
\hline 7 & Not considered success and failure of treatment. & Success and failure of treatment is considered. \\
\hline 8 & $\begin{array}{l}\text { We have fifth compartment which contains no human population. It } \\
\text { contains environmental pathogen population. }\end{array}$ & $\begin{array}{l}\text { We have fifth compartment which contains no human population. } \\
\text { It contains environmental pathogen population. }\end{array}$ \\
\hline 9 & $\begin{array}{l}\text { One equilibrium point was analyzed. That is disease free equilibrium } \\
\text { point. }\end{array}$ & $\begin{array}{l}\text { Three equilibrium points were analyzed. Disease - free equilibrium } \\
\text { point, endemic equilibrium point and epidemic equilibrium point. }\end{array}$ \\
\hline \multirow[t]{2}{*}{10} & $\begin{array}{l}\text { Reproduction ratio is slower whose formula is } \\
\qquad \alpha \in \Lambda \beta\end{array}$ & $\begin{array}{l}\text { Reproduction ratio is faster whose formula is } \\
\qquad \alpha \in \Lambda \beta\end{array}$ \\
\hline & $R_{0}=\overline{A c \mu \mu_{0}(\beta+\mu)(\delta+\mu+d)}$ & ${ }_{0}=\overline{A c \mu \mu_{0}(\beta+\mu)(\delta+\mu+d)-r \beta \alpha}$ \\
\hline 11 & $\begin{array}{l}\text { The more important parameter to control the spread of TB is to reduce the } \\
\text { crowded areas in which the people live. }\end{array}$ & $\begin{array}{l}\text { The more important parameter to control the spread of TB is to } \\
\text { reduce the crowded areas in which the people live. }\end{array}$ \\
\hline 12 & Nil & $\begin{array}{l}\text { Taking medications as often reduce the multi - drug resistance } \\
\text { tuberculosis. }\end{array}$ \\
\hline
\end{tabular}

\section{Conclusions}

From numerical simulation we can summarize that the most important parameter is the area in which the people live. When the spatial area $A$ is increased a little in size then both the reproduction ratio $R_{0}$ and probability of catching tuberculosis due to polluted air $\lambda$ decrease to lower values. That is $A$ is inversely proportional to both $R_{0}$ and $\lambda$. If people lives together in very crowded area then the spread of tuberculosis disease increases. Therefore by diluting the crowded areas we can control the spread of tuberculosis. Completing the treatment and taking medications as often reduce the multi drug resistance (MDR) tuberculosis mortality and incidence rates.

From the results of the stability analysis, we have shown that the disease free equilibrium point is asymptotically stable while the endemic and epidemic equilibrium points are unstable. Whether the disease becomes persistent or dies out depends on the magnitude of the basic reproductive number $R_{0}$. We found that if the basic reproduction ratio $R_{0}<1$, then each solution leads to the disease free equilibrium point. In other words, every infectious individual will cause less than one secondary infection. Hence the disease dies out after some time. If $R_{0}>1$ then there exist a unique epidemic equilibrium point. This means that one infected person can transmit disease for more than one person and tuberculosis disease spread continuous in the society. If $R_{0}=1$ then there exist a unique endemic equilibrium point. Meaning is that the disease remains in the population at a consistent level, as one infected individual transmits the disease to one susceptible.

\section{References}

[1] Dancho Desalegn and Purnachandra Rao Koya. The Role of polluted air and population density in the spread of Mycobacterium tuberculosis disease. Journal of Multidisciplinary Engineering Science and Technology (JMEST), Vol. 2 Issue 5, May - 2015. http://www.jmest.org/wpcontent/uploads/JMESTN42350782.pdf.

[2] Dr. Maria Sanchez, Lecture 9: Tuberculosis pdf UC Berkeley Ongoing TB and HIV research.

[3] Hongbin Guo and Michael Y. Li. Global stability in a Mathematical Model of Tuberculosis. Canadian Applied Mathematics Quarterly, Volume 14, Number 2, Summer 2006.

[4] PHAC. "Tuberculosis FACT SHEETS." 2008. http://www.phac-aspc.gc.ca/tbpclatb/fa-fi/trans-eng.php.

[5] Global Tuberculosis Report 2012. World Health Organization 20 Avenue Appia, 1211-Geneva-27, Switzerland Email: tbdocs@who.int Web site: www.who.int/tb.

[6] Global tuberculosis report 2013. World Health Organization. Geneva: 23 Oct 2013. Available from: http://apps.who.int/iris/bitstream/10665/91355/1/9789241564 656-eng.pdf.

[7] World Health Organization (WHO). Global tuberculosis report 2014.

[8] WHO. Fact sheet number 104. Technical report, World Health Organization, Geneva, Switzerland, 2012.

[9] The patient education institute 1995 - 2012, Inc.www. $X$ plain.come last reviewed: 10/15/2012. 
[10] Dejen Ketema Mamo and Purnachandra Rao Koya. Mathematical Modeling and Simulation Study of SEIR disease and Data Fitting of Ebola Epidemic in West Africa. Journal of Multidisciplinary Engineering Science and Technology (JMEST). Vol. 2, Issue 1, January 2015, pp 106 - 14. ISSN: $3159 \quad-\quad 0040 . \quad$ http://www.jmest.org/wpcontent/uploads/JMESTN42350340.pdf.

[11] Purnachandra Rao Koya and Dejen Ketema Mamo. Ebola Epidemic Disease: Modelling, Stability Analysis, Spread Control Technique, Simulation Study and Data Fitting. Journal of Multidisciplinary Engineering Science and Technology (JMEST). Vol. 2, Issue 3, March 2015, pp 476 - 84. ISSN: $3159 \quad-\quad 0040 . \quad \mathrm{http}: / / \mathrm{www} . j m e s t . o r g / \mathrm{wp}-$ content/uploads/JMESTN42350548.pdf.

[12] Abdulsamad Engida Sado and Purnachandra Rao Koya. Application of Brody Growth Function to Describe Dynamics of Breast Cancer Cells. American Journal of Applied Mathematics (AJAM). Vol. 3, No. 3, 2015, pp. 138-145. Doi:10.11648/j.ajam.20150303.20.

[13] Fekadu Tadege Kobe and Purnachandra Rao Koya. Controlling the Spread of Malaria Disease Using Intervention Strategies. Journal of Multidisciplinary Engineering Science and Technology (JMEST). Vol. 2, Issue 5, May 2015, pp 1068 - 74. ISSN: 3159 - 0040. http://www.jmest.org/wpcontent/uploads/JMESTN42350745.pdf.

[14] WHO. "Tuberculosis (TB)", 2011. http://www.who.int/tb/en

[15] S. O. Adewale, C.N. Podder and A. B. Gumel, Mathematical analysis of a TB transmission model with DOTS. Canadian Applied mathematics Quarterly, Volume 17, Number 1, spring 2009.

[16] P. van den Driessche and James Watmough, Reproduction numbers and sub-threshold endemic equilibria for compartmental models of disease transmission. Mathematical Biosciences 180 (2002) 29-48. www.elsevier.com/locate/mbs.

[17] A. Ssematimba J. Y. T., Mugisha and L. S. Luboobi, Mathematical Models for the Dynamics of Tuberculosis in Density-dependent Populations: The Case of Internally Displaced Peoples' Camps (IDPCs) in Uganda. Journal of Mathematics and Statistics 1(3): 217-224, 2005.

[18] S. Marino and D. Kirschner, The human immune response to the Mycobacterium tuberculosis in lung and lymph node, J. Theor. Biol., 227 (2004), 463-486. 